\title{
Études préliminaires de la distribution spatio-temporelle du phytoplancton dans un système fluvio-lacustre africain (Bassin Bia ; Côte d'Ivoire)
}

\section{Preliminary studies of spatio-temporal distribution of phytoplankton in an africain stream-lake system (Bia basin; Côte d'Ivoire)}

\author{
A. Ouattara ${ }^{(1) *}$, N. Podoor ${ }^{(2)}$ et G. Gourène ${ }^{(1)}$ \\ (1) Laboratoire d'Environnement et de Biologie Aquatique, Université d'Abobo-Adjamé 02 \\ BP 801 Abidjan 02, Côte d'lvoire. \\ *E.mail : allassane ouattara@hotmail.com \\ (2) Katholieke Universiteit Leuven, Instituut voor Plantkunde, Morphologie, Systematiek en \\ Ecologie der planten, Kardinaal Mercierlaan 92, B-3001 Leuven, Belgique.
}

Résumé - Les échantillons examinés mensuellement de novembre 1996 à octobre 1997 proviennent de divers endroits du système fluvio-lacustre de la Bia : deux sites dans le lac (Bakro et Ayamé) et deux dans le système fluvial dont un en amont (Bianouan) et un en aval (Aboisso).

La structure et la dynamique du phytoplancton ont été appréciées. En amont du barrage, le peuplement est marqué, presque toute l'année, par une relative abondance des Diatomées Aulacoseira granulata et Fragilaria virescens. Dans le lac, l'évolution du peuplement phytoplanctonique est plutôt fonction des deux grandes saisons de l'année. Les Diatomées, principalement, Asterionella formosa, Aulacoseira granulata et A. granulata var. angustissima dominent le peuplement en saison sèche. Pendant les périodes pluvieuses de l'année, les Cyanophycées Microcystis aeruginosa. Anabaena spiroides constituent la composante majoritaire du phytoplancton dans les échantillons. En aval du barrage hydroélectrique, la dynamique du phytoplancton observée est plus ou moins semblable à celle ci-dessus décrite dans le systeme lacustre.

Les variations des paramètres physico-chimiques enregistrées dans les différentes stations semblent peu influencer la densité du phytoplancton. Elle apparaît plutôt déterminée par le régime hydrologique de la rivière.

Mots clés - Phytoplancton, Distribution, Système fluvio-lacustre, Côte d'Ivoire.

Abstract - The examined samples carried out monthly from november 1996 to october 1997 come from various places of the river Bia: two locations in the lake (Bakro and Ayamé) and one in upstream (Bianouan) and the other one in downstream (Aboisso). 
The structure and the dynamic of the phytoplankton have been appreciated. Upstream of the dam, the population is dominated, nearly all year round, by Diatoms, Aulacoseira granulata and Fragilaria virescens. In the lake, the evolution of phytoplankton is rather function of the two big seasons of the year. Diatoms, mainly, Asterionella formosa, Aulacoseira granulata and $A$. granulata var. angustissima are abundant among phytoplankton population in dry season. During the rainy periods, Cyanophyceae, Microcystis aeruginosa and Anabaena spiroides constituted the majority taxa of the phytoplankton. Downstream the hydroelectric dam, the dynamic of the phytoplankton is more similar to those of the lake.

Physical and chemical parameter variations recorded in the different locations do not influence very well the density of the phytoplankton. This density seem to be determined by hydrological regime of the river.

Keyword - Phytoplankton, Distribution, Stream-lake System, Côte d'Ivoire.

\section{INTRODUCTION}

L'étude des algues phytoplanctoniques est fondamentale dans les recherches environnementales relatives aux écosystèmes aquatiques. Les algues phytoplanctoniques sont souvent exploitées pour apprécier la qualité des eaux (Carlson, 1977). Pour Dufour \& Durand (1982), ces organismes occupent dans le milieu aquatique une position trophique importante et en constituent, de ce fait, un élément déterminant de leur fonctionnement.

La Bia est un petit bassin côtier situé à l'extrême Sud-est ivoirien dont la surface du bassin versant est de $9650 \mathrm{~km}^{2}$ (Van den Bossche \& Bernacsek, 1990) (fig. 1). Son régime hydrologique a d'abord été modifié en 1959 par la construction d'un premier barrage hydroélectrique (Ayamé I) et d'un second (Ayamé II) en 1965, 4 km en aval du premier.

Le réservoir créé par le barrage Ayamé l est devenu le premier centre véritable de la pêche en Côte d'Ivoire
(Gourène et al., 1999). C'est vraisemblablement à ce titre que de nombreuses études y ont été consacrées. Les seules données relatives aux algues phytoplanctoniques portant sur le bassin de la Bia se résument à un inventaire de Ouattara et al., (2000).

Aussi, la présente étude se propose-t-elle d'apprécier la dynamique du phytoplancton dans ce système fluvio-lacustre dans l'optique d'une contribution à une meilleure compréhension de son fonctionnement.

\section{MATÉRIEL ET MÉTHODES}

\subsection{Sites de prélèvement}

Quatre stations ont été retenues et prospectées mensuellement de novembre 1996 à octobre 1997 sur le systeme fluvio-lacustre de la Bia (fig. 1): deux dans le lac d'Ayamé 1 (Bakro et Ayamé) et deux dans le bassin fluvial dont un en amont (Bianouan) et un en aval (Aboisso). 


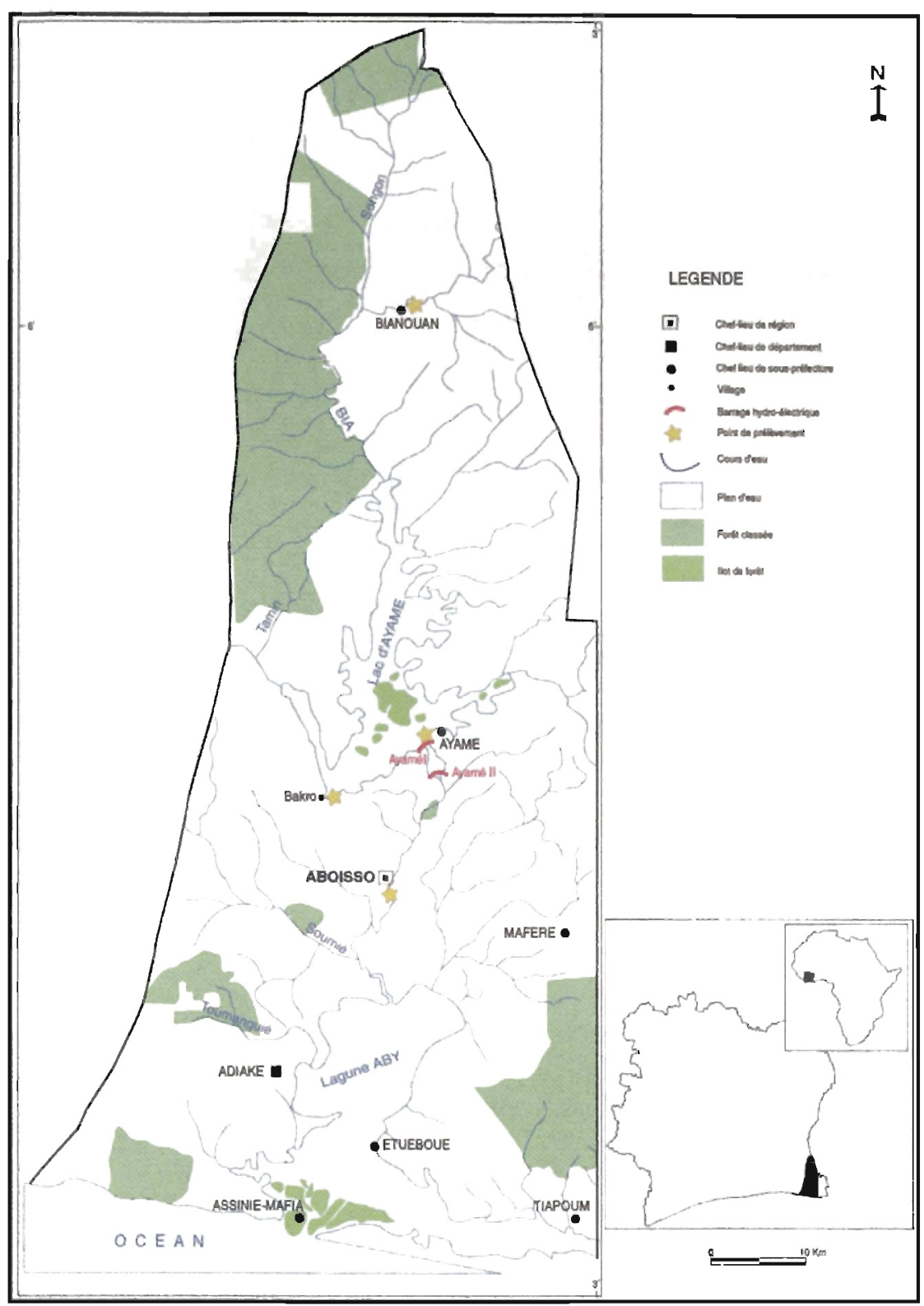

Fig. 1 - Situation géographique de la rivière Bia et localisation des stations de prélèvement (Source : Centre National de Télédétection et d'Information Géographique, modifiée par Apex Infor Communication).

Fig. 1 - Geographical location of the Bia river and sampling stations (from National Teledetection Center and Geography Information modified by Apex Infor communication). 


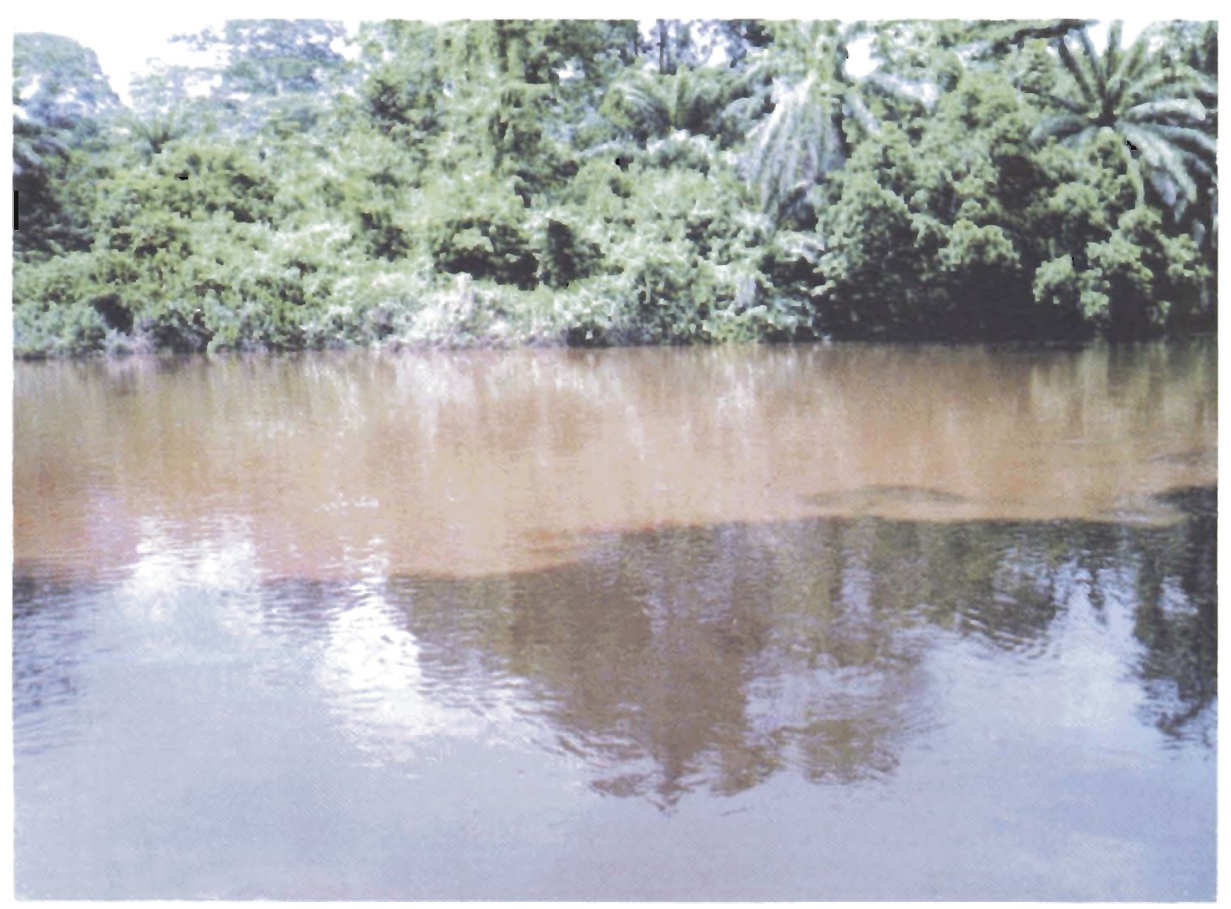

Planche photographique 1A - Vue du plan d'eau en amont des barrages.

Photographic plate $1 \mathrm{~A}$ - View of the upstream.

La station de Bianouan $\left(6^{\circ} 01^{\prime} \mathrm{N}\right.$ et $3^{\circ} 11^{\prime} W$ ) en amont du barrage hydroélectrique, présente une rive majoritairement boisée (planche 1A).

Le substrat est principalement constitué de gros galets au centre du lit. La cote est maximale en juillet $(8,5 \mathrm{~m})$ et minimale en février $(1,5 \mathrm{~m})$. Les largeurs de la rivière Bia à cette station se situent entre 15 et $40 \mathrm{~m}$ ( $25 \mathrm{~m}$ en moyenne) et les profondeurs entre $0,5 \mathrm{~m}$ et $8,5 \mathrm{~m}$ ( $3 \mathrm{~m}$ en moyenne). En zone entièrement villageoise, cette station est encore préservée d'activités anthropiques.
Le barrage d'Ayamé I est caractérisé par une hauteur de hors sol de $30 \mathrm{~m}$ et d'un développement en crête de $575 \mathrm{~m}$. Son réservoir occupe une superficie de $190 \mathrm{~km}^{2}$ pour une largeur moyenne de $27 \mathrm{~km}$ et une longueur moyenne de $80 \mathrm{~km}$. II permet de retenir près de 1 milliard de $\mathrm{m}^{3}$ d'eau en période de crue (source Compagnie Ivoirienne d'Electricité). A l'inverse, le barrage d'Ayamé II présente des caractéristiques plus modestes: $30 \mathrm{~m}$ de hauteur, $310 \mathrm{~m}$ de longueur de crête, $1 \mathrm{~km}^{2}$ de superficie pour 5 millions de $\mathrm{m}^{3}$ d'eau de retenue. 


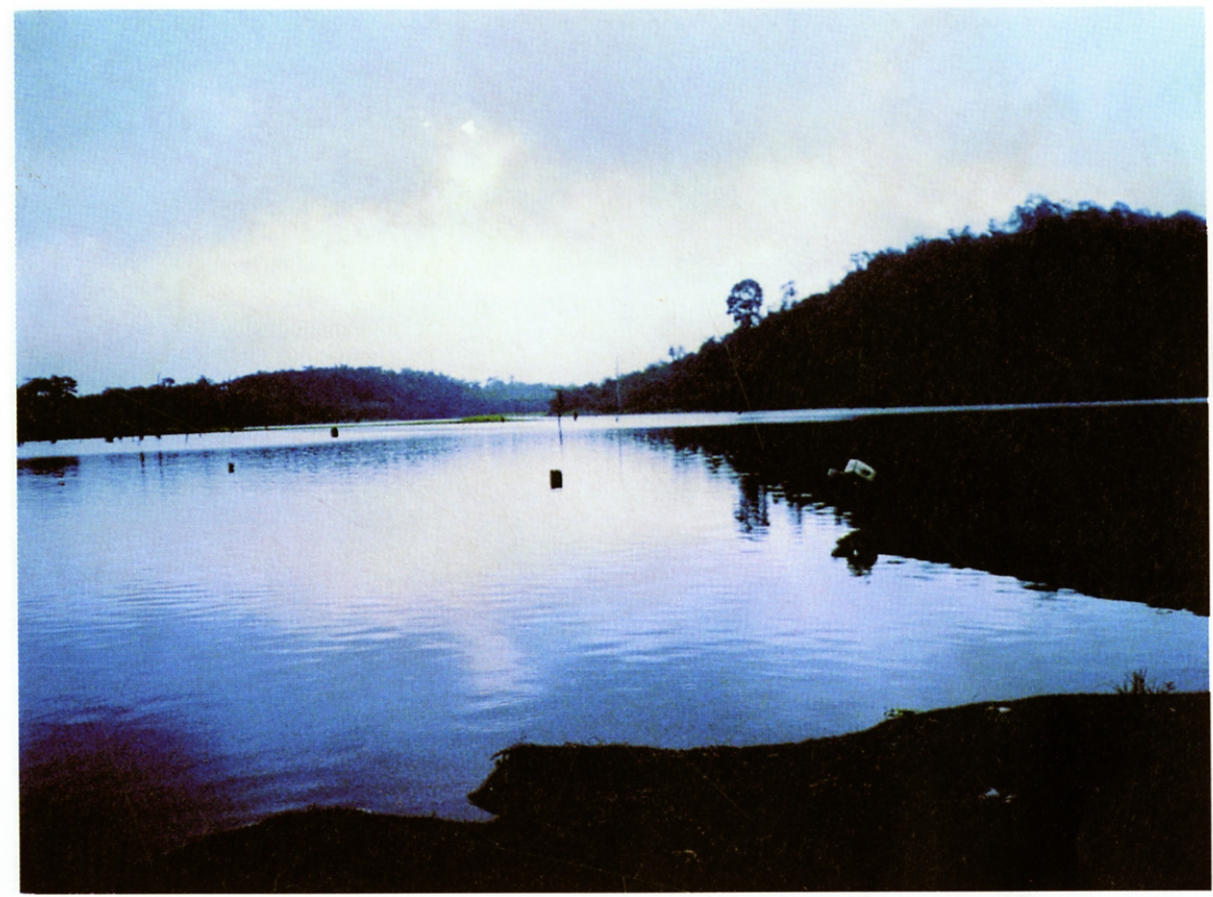

Planche photographique 1B - Vue du lac à la station d'Ayamé.

Photographic plate 1B - View of the lake at Ayamé.

De l'étiage aux plus hautes eaux, les cotes enregistrées dans le lac à la station d'Ayamé varient de $84,84 \mathrm{~m}$ à $88,44 \mathrm{~m}$ (87,5 m en moyenne). La production hydroélectrique est assurée par les apports de la rivière Bia. Les apports les plus importants ont été notés en juin (491,41 millions de $\mathrm{m}^{3}$ ) et les plus faibles en février $\left(0,88\right.$ millions de $\left.\mathrm{m}^{3}\right)$. Le débit moyen annuel de ces apports au cours de notre échantillonnage est de $44,59 \mathrm{~m}^{3} / \mathrm{s}$ (Source : Compagnie Ivoirienne d'Electricité).

Dans le lac d'Ayamé I, la station de Bakro $\left(5^{\circ} 33^{\prime} \mathrm{N}\right.$ et $\left.3^{\circ} 15^{\prime} \mathrm{W}\right)$ pré- sente des hauteurs d'eau relativement faibles (10 $\mathrm{m}$ en moyenne). Quant à la station d'Ayamé ( $5^{\circ} 36^{\prime} \mathrm{N}$ et $\left.3^{\circ} 10^{\prime} \mathrm{W}\right)$, au pied du barrage du même nom (planche 1B), la profondeur moyenne est plus importante (15 m en moyenne).

Ces deux stations sont peu affectées par les rejets d'eaux usées mais sont le siège d'une intense activité agricole.

Le site de prélèvement dans la ville d'Aboisso ( $5^{\circ} 28^{\prime} \mathrm{N}$ et $3^{\circ} 12^{\prime} \mathrm{W}$ ) est situé en aval du barrage hydroélectrique (planche 1C). 


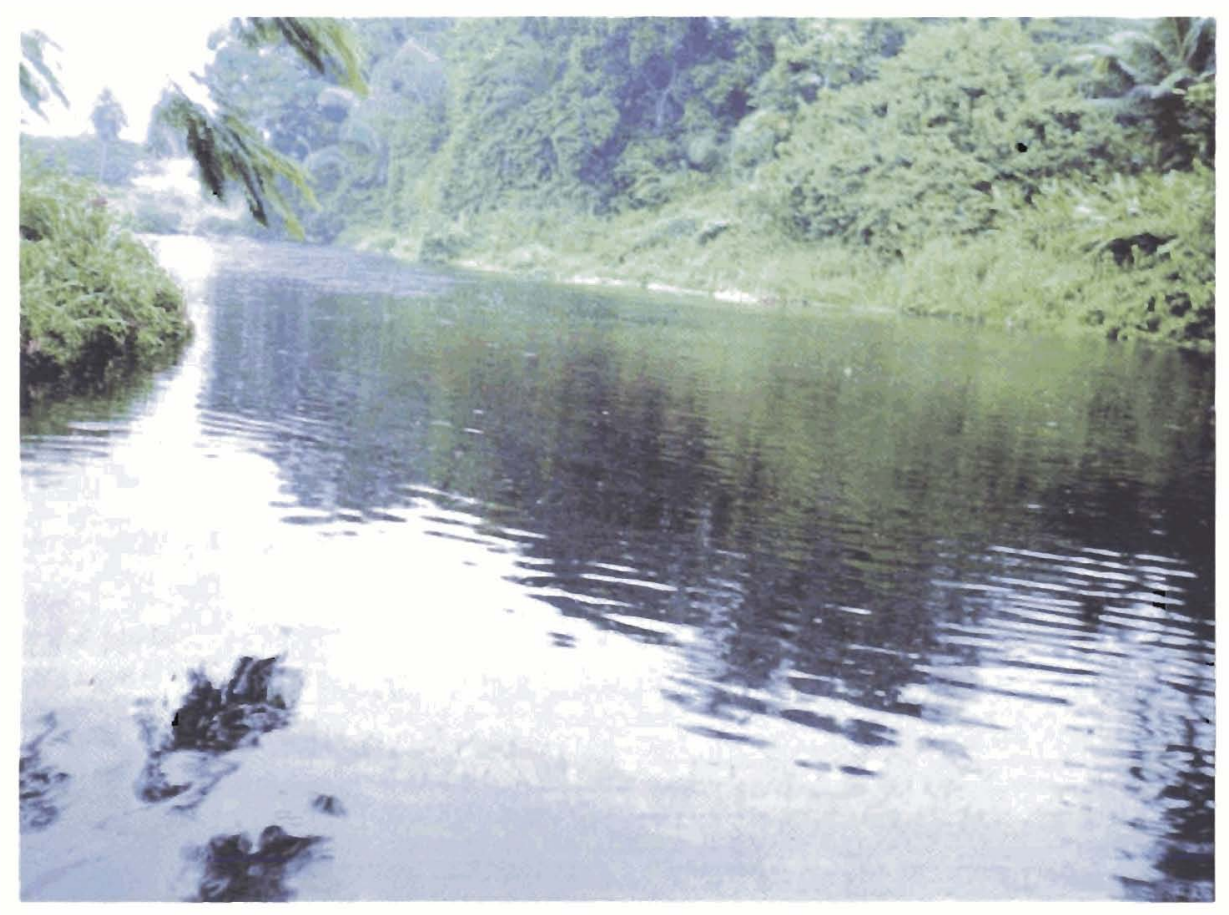

Planche photographique $1 \mathrm{C}$ - Vue du plan d'eau en aval des barrages (Cốte d'Ivoire). Photographic plate 1C - View of the downstream (Cóte d'Ivoire).

Les caractéristiques hydrologiques sont constamment modifiées par la gestion des turbines des barrages Ayamé I et II. Les largeurs varient entre $30 \mathrm{~m}$ et $70 \mathrm{~m} \quad(40 \mathrm{~m}$ en moyenne) et les profondeurs entre 3 et 5,7 m (4,3 m en moyenne). Les cotes n'ont pu être notées par manque de station de relevé. Les eaux de la rivibre à cette station sont affectées par de nombreuses activités humaines (baignade, lessive, rejets domestiques des agglomérations).

Le climat de la région d'étude est caractérisé par deux saisons sèches et deux saisons de pluies. La pro- miére saison sèche généralement plus longue va de décembre à mars contre août à septembre pour la petite saison sèche. La grande saison de pluies est centrée sur juin et la petite sur octobre. En période pluvieuse, les eaux de ruissellement, qui participent à l'érosion du bassin versant, amènent des matières en suspension dans la rivière Bia. Le régime hydrologique du système fluvio-lacustre de la Bia est plus ou moins lié à celui des précipitations (fig. 2). La Bia reçoit différents affluents dont Songan, Tamin et Soumié (fig. 1). 

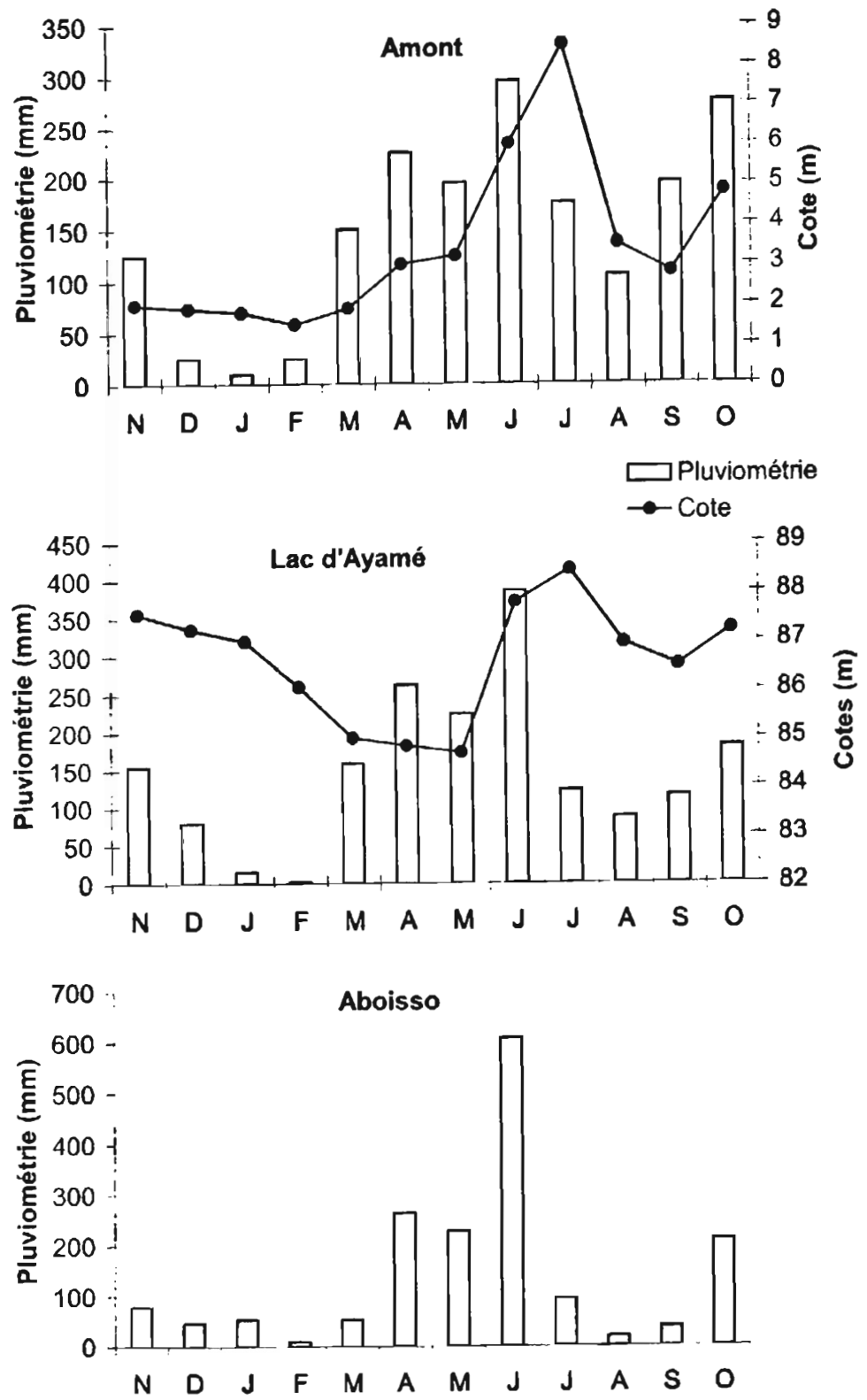

Fig. 2 - Variation de la pluviométrie dans les différentes stations (amont, aval et Ayamé) et du niveau des eaux en amont et à Ayamé de novembre 1996 à octobre 1997 (Source : Compagnie Ivoirienne d'Électricité (CIE) et Sociètè d'Exploitation de l'Aviation et de la Météorologie (SODEXAM).

Fig. 2 - Variation of rainfall at every sampling location (upstream, downstream, Ayamé) and water level at upstream and Ayamé for november 1996 to october 1997. 
La forêt du bassin versant est caractérisée par quelques associations de Khaya ivorensis, Dumoria heckelii, Maponia sp. et de Diospyros sp. (Reizer, 1967). Cette zone supporte en plus des cultures vivrières (manioc...), des plantations industrielles et villageoises de palmiers à huile, d'ananas, de bananes, de café et de cacao.

\section{2 Échantillonnage et techniques d'analyses}

Les prélèvements relatifs à cette étude ont été effectués mensuellement de novembre 1996 à octobre 1997. Les quatre stations retenues ont été échantillonnées en surface $(40 \mathrm{~cm})$ à l'aide d'une bouteille hydrologique de Van Dorn.

Les comptages phytoplanctoniques ont été réalisés à l'aide d'un microscope Zetopan-Reichert du type Autriche à partir d'aliquotes des différents échantillons fixés au lugol. Le dénombrement cellulaire de chaque échantillon exprimé en nombre de cellules par litre a été fait, entre lame et lamelle, sur une suite de champs optiques. La détermination préliminaire du nombre de montages à dénombrer, en fonction de la dispersion aléatoire du phytoplancton dans l'échantillon homogénéisé, suit la méthodologie de Uehlinger (1964) reprise par Lazzaro (1981).

Les mesures d'oxygène dissous $\left(\mathrm{O}_{2}\right)$ et de température de l'eau (oxymètre OXI 96), de la conductivité et du taux de solides dissous (conducti- mètre LF95), de $\mathrm{pH}$ (pHmètre WTW) et de transparence (disque de Seechi), ont été effectuées in situ entre 7 heures et 8 heures, excepté la transparence (10 heures). Le dosage des anions $\left(\mathrm{NO}_{2}, \mathrm{NO}_{3}, \mathrm{P}-\mathrm{PO}_{1}{ }^{3-}\right)$ et des cations $\left(\mathrm{NH}_{1}{ }^{\prime}, \mathrm{Ca}^{2 \cdot}\right)$, de la dureté totale (DT), a été fait à l'aide de la trousse HACH DR 2000 sur des échantillons d'eau prélevés en surface.

\subsection{Analyse statistique}

Le logiciel statistique utilisé est le CANOCO (Ordination Canonique des Communautés, version 4.0) (Ter Braak \& Smilauer, 1998). Ce programme a permis dans un premier temps, à partir d'une analyse DCA (Analyse des Correspondances "Détendancées"), portant sur les densités des taxons, de déterminer la longueur du gradient en unité de déviation standard (SD). Lorsque le maximum de longueur du gradient n'excède pas $4 \mathrm{SD}$, la méthode linéaire RDA (Analyse de ReDondance) est préférable aux méthodes unimodales CCA (Analyse Canonique des Correspondances) et DCCA (Analyse Canonique des Correspondances "Détendancées"). Ainsi, l'analyse RDA, technique directe d'analyse multivariée éprouvée pour le traitement des données floristiques et écologiques (Muylaert et al., 1997 ; Van Tongeren et al., 1992), a été appliquée. C'est une méthode qui permet une interprétation aisée des ordinations par une analyse simultanée des variables abiotiques et bio- 
tiques. L'analyse a été effectuée à partir des abondances relatives des taxons phytoplanctoniques présentant un pourcentage supérieur à $5 \%$ de la densité totale dans chaque milieu. Ces données ont subi une transformation logarithmique $[\log (x+1)]$.

\section{RÉSULTATS}

\subsection{Variables abiotiques}

Les valeurs moyennes et les écart-types des variables physico-chimiques relatifs à chaque station d'échantillonnage sont représentés sur la Figure 3.

Les coefficients de corrélation entre les différentes variables, extraits de la matrice de corrélation de l'analyse en RDA, sont donnés dans les tableaux I et II.

Les températures enregistrées dans les stations du lac (Bakro, Ayamé) sont plus élevées que celles observées en amont et en aval du barrage. Les valeurs moyennes de $\mathrm{pH}$ relevées dans l'ensemble des stations sont voisines de 7,4.

Tout comme la température, les valeurs de la transparence sont plus importantes dans les stations du lac (Bakro, Ayamé) que celles enregistrées en amont et en aval du barrage. Quant au taux d'oxygène dissous, il est en moyenne un peu plus élevé en aval du barrage que dans les autres stations étudiées. Les données de la conductivité (CND), du taux de solides dissous (TDS), du calcium $\left(\mathrm{Ca}^{2+}\right.$ ) et de la dureté totale (DT) obtenues en amont du barrage, sont relativement plus fortes que celles des stations du lac (Bakro, Ayamé) et de l'aval. Les teneurs en phosphates $\left(\mathrm{P}-\mathrm{PO}_{4}{ }^{3-}\right)$ sont également plus importantes en amont que dans les autres stations de prélèvements de la rivière.

Les concentrations moyennes en nitrates $\left(\mathrm{NO}_{3}^{-}\right)$, nitrites $\left(\mathrm{NO}_{2}\right)$ et en ammonium $\left(\mathrm{NH}_{4}{ }^{+}\right)$enregistrées, varient très peu d'une station à une autre.

\subsection{Densité phytoplanctonique}

Les grands groupes taxonomiques recensés sont les Chlorophytes (Chlorophycées et Conjugatophycées), Diatomées, Cyanophycées, Euglénophycées, Dinophycées, Xanthophycées et Chrysophycées. Les pourcentages des deux derniers groupes n'excédant pas $1 \%$ de la densité totale enregistrée dans chaque station, aussi n'ont-ils pas été pris en compte dans l'élaboration des différentes illustrations graphiques.

En amont du barrage, la densité absolue du phytoplancton présente sa valeur maximale en novembre pendant la petite saison de pluies (fig. 4).

Elle décroît ensuite progressivement jusqu'en février où elle atteint son premier minimum. De mars à juin, cette densité reste peu élevée. En août, on observe le second minimum suivi d'un accroissement de la densité de septembre à octobre. 

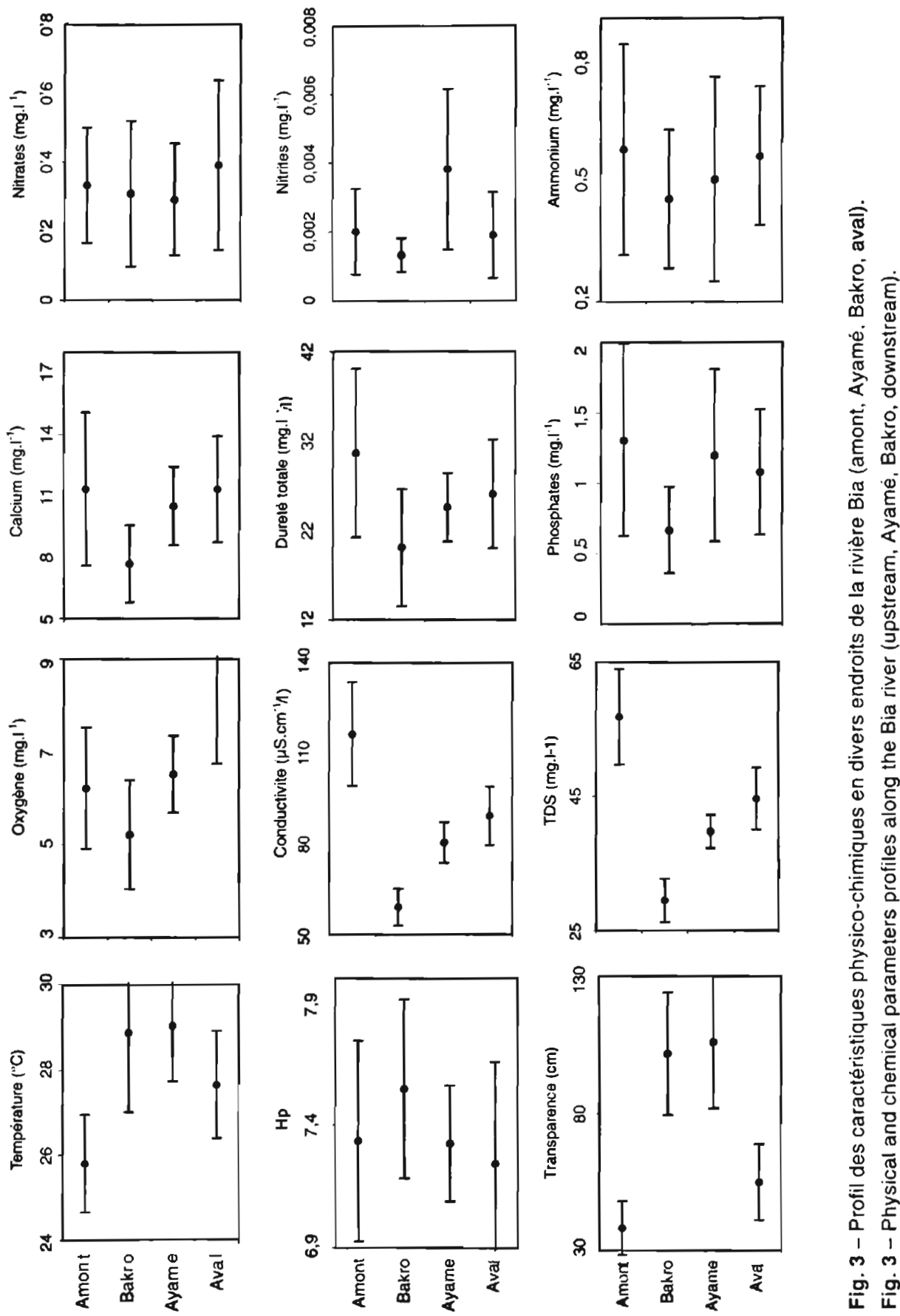
Tableau I - Coefficients de corrélation entre les variables physico-chimiques en amont et en aval des barrages hydro-électrique de novembre 1996 à octobre 1997.

Table I - Coefficients of correlation between differents physical and chemical parameters at upstream and downstream the hydroelectric dams for november 1996 to october 1997.

\begin{tabular}{|c|c|c|c|c|c|c|c|c|c|c|c|c|c|}
\hline \multirow{15}{*}{ Amont } & & $T^{\circ}$ eau & $T^{\circ}$ air & pH & CND & TDS & 02 & Transp. & Prof. & Pluvio. & PO4 & $\mathrm{Ca} 2+$ & DT \\
\hline & Moyenne & 25,8 & 26,4 & 7,3 & 116,3 & 56,8 & 6,2 & 38,3 & 3,7 & 149,9 & 1,3 & 11,3 & 30,7 \\
\hline & Ecart type & 1,14 & 1,71 & 0,41 & 17,17 & 7,13 & 1,31 & 9,79 & 2,63 & 120,82 & 0,68 & 3,75 & 9,42 \\
\hline & $T^{\circ}$ eau & 1 & & & & & & & & & & & \\
\hline & $\mathbf{T}^{\circ}$ air & 0,845 & 1 & & & & & & & & & & \\
\hline & pH & $-0,459$ & $-0,665$ & 1 & & & & & & & & & \\
\hline & CND & 0,649 & 0,584 & $-0,362$ & 1 & & & & & & & & \\
\hline & TDS & 0,563 & 0,331 & $-0,110$ & 0,851 & 1 & & & & & & & \\
\hline & $\mathrm{O} 2$ & 0,304 & 0,312 & $-0,622$ & 0,329 & 0,318 & 1 & & & & & & \\
\hline & Transp. & 0,476 & 0,637 & $-0,717$ & 0,345 & 0,229 & 0,423 & 1 & & & & & \\
\hline & Prof. & $-0,410$ & $-0,341$ & 0,031 & $-0,581$ & $-0,449$ & $-0,236$ & $-0,147$ & 1 & & & & \\
\hline & Pluvio. & $-0,255$ & $-0,321$ & 0,135 & $-0,589$ & $-0,481$ & $-0,295$ & $-0,368$ & 0,804 & 1 & & & \\
\hline & PO4 & $-0,066$ & $-0,127$ & 0,176 & $-0,292$ & $-0,360$ & $-0,588$ & -0.434 & 0,193 & 0,440 & 1 & & \\
\hline & Ca2.+ & $-0,188$ & $-0,029$ & $-0,031$ & 0,239 & 0,323 & 0,026 & $-0,243$ & $-0,086$ & $-0,186$ & 0,000 & 1 & \\
\hline & DT & $-0,072$ & $-0,041$ & $-0,137$ & 0,229 & 0,277 & 0.515 & -0.040 & $-0,144$ & $-0,180$ & $-0,320$ & 0,477 & 1 \\
\hline \multirow{15}{*}{ Aval } & & T'eau & $\mathbf{T}^{\circ}$ air & $\mathrm{pH}$ & CND & TDS & 02 & Transp. & Prof. & Pluvio. & PO4 & Ca2+ & DT \\
\hline & Moyenne & 27,6 & 28,6 & 7,2 & 89,2 & 44,7 & 9,1 & 54.9 & 4,3 & 149.1 & 1,1 & 11,3 & 26,1 \\
\hline & Ecart type & 1,26 & 1,56 & 0,42 & 9,64 & 4,59 & 2,38 & 13,80 & 1,36 & 168,35 & 0,45 & 2,61 & 6,07 \\
\hline & $\mathbf{T}^{\circ}$ eau & 1 & & & & & & & & & & & \\
\hline & $\mathbf{T}^{\circ}$ air & 0,772 & 1 & & & & & & & & & & \\
\hline & pH & $-0,638$ & $-0,510$ & 1 & & & & & & & & & \\
\hline & CND & 0,392 & 0,296 & $-0,481$ & 1 & & & & & & & & \\
\hline & TDS & 0,380 & 0,317 & $-0,533$ & 0,976 & 1 & & & & & & & \\
\hline & 02 & $-0,091$ & $-0,428$ & 0,197 & $-0,125$ & $-0,131$ & 1 & & & & & & \\
\hline & Transp. & 0,390 & $-0,064$ & $-0,385$ & 0,326 & 0,369 & 0,172 & 1 & & & & & \\
\hline & Prot. & $-0,311$ & 0,000 & 0,776 & $-0,439$ & $-0,458$ & 0,014 & $-0,444$ & 1 & & & & \\
\hline & Pluvio. & 0,343 & 0,672 & $-0,076$ & 0,012 & 0,077 & $-0,116$ & $-0,191$ & 0,457 & 1 & & & \\
\hline & PO4 & $-0,017$ & $-0,449$ & 0,154 & $-0,006$ & $-0,058$ & 0,578 & 0,114 & $-0,359$ & $-0,569$ & 1 & & \\
\hline & $\mathrm{Ca2+}$ & -0.174 & $-0,202$ & 0,497 & $-0,440$ & $-0,517$ & 0,062 & $-0,507$ & 0,442 & $-0,249$ & 0,244 & 1 & \\
\hline & DT & $-0,080$ & $-0,365$ & 0,410 & $-0,415$ & $-0,374$ & 0,506 & 0,054 & 0,271 & $-0,147$ & 0,525 & 0,5789 & 1 \\
\hline
\end{tabular}


Tableau II - Coefficients de corrélation entre les variables physico-chimiques dans le lac d'Ayamé I (Bakro, Ayamé) de novembre 1996 à octobre 1997.

Table II - Coefficients of correlation between differents physical and chemical parameters at Ayamé I lake (Bakro, Ayamé) for november 1996 to octuber 1997.

\begin{tabular}{|c|c|c|c|c|c|c|c|c|c|c|c|c|c|}
\hline \multirow[t]{15}{*}{ ! } & \multirow{2}{*}{ '- Movenne } & T"eau & Toair & \multicolumn{2}{|c|}{$\mathrm{pH} \quad \mathrm{CND}$} & TOS & \multicolumn{2}{|c|}{$02 \quad$ Transp. } & Prof. & Pluvio. & $\mathrm{PO4}$ & $\mathrm{Ca2+}$ & DT \\
\hline & & 28.9 & 29,0 & $7, \overline{5}$ & $\overline{59.0}$ & 29,4 & $5, \overline{2}$ & 101.7 & 9,7 & 149.6 & 0.7 & 7.7 & 20,1 \\
\hline & Ecart type & 1,86 & 2,32 & 0,37 & 6.07 & 3,19 & 1,18 & 22,33 & 2,76 & 107,01 & 0,31 & 1,87 & 6,56 \\
\hline & T"eau & 1 & & & & & & & & & & & \\
\hline & $T^{\top}$ air & 0,355 & 1 & & & & & & & & & & \\
\hline & $\mathrm{pH}$ & -0.258 & 0,445 & 1 & & & & & & & & & \\
\hline & CND & 0.305 & $-0,239$ & $-0,235$ & 1 & & & & & & & & \\
\hline & TDS & 0,351 & $-0,231$ & $-0,291$ & 0,995 & 1 & & & & & & & \\
\hline & 02 & 0,429 & 0,376 & 0,091 & 0.248 & 0,242 & 1 & & & & & & \\
\hline & Transp. & $-0,151$ & $-0,711$ & $-0,165$ & 0.306 & 0,283 & $-0,516$ & 1 & & & & & \\
\hline & Prof. & 0.552 & 0,639 & -0.055 & $-0,273$ & -0.234 & 0,575 & $-0,794$ & 1 & & & & \\
\hline & Pluvio. & 0.407 & 0,641 & $-0,077$ & $-0,347$ & -0.306 & 0,593 & $-0,908$ & 0,934 & 1 & & & \\
\hline & PO4 & $-0,258$ & 0,445 & 1,000 & $-0,235$ & $-0,291$ & 0,091 & $-0,165$ & $-0,055$ & $-0,077$ & 1 & & \\
\hline & $\mathrm{Ca} 2+$ & $-0,212$ & 0.274 & 0,392 & 0.081 & 0.090 & 0,028 & $-0,224$ & $-0,101$ & 0,001 & 0,392 & 1 & \\
\hline & DT & -0.117 & -0.396 & -0.244 & 0,498 & 0,508 & -0.200 & 0.245 & $-0,523$ & $-0,385$ & $-0,244$ & 0,402 & 1 \\
\hline \multirow{15}{*}{ Ayamé } & & Toeau & $\mathrm{T}^{\circ}$ aìr & $\mathrm{pH}$ & CND & TDS & 02 & Transp. & Prof. & Pluvio. & $\mathrm{PO} 4$ & $\mathrm{Ca} 2+$ & DT \\
\hline & Moyenne & 29.0 & 29,1 & 7,3 & 80,4 & 39,7 & $\overline{6}, \overline{5}$ & 105.9 & 13,4 & 149,6 & 1,2 & 10,5 & 24,6 \\
\hline & Ecart tyype & 1,31 & 1,92 & 0,24 & 6,75 & 2,48 & 0,82 & 24,19 & 2,53 & 107,01 & 0,61 & 1,93 & 3.85 \\
\hline & T"eau & 1 & & & & & & & & & & & \\
\hline & Tªir & 0,803 & 1 & & & & & & & & & & \\
\hline & $\mathrm{pH}$ & -0.095 & -0.070 & 1 & & & & & & & & & \\
\hline & CND & 0,392 & 0,309 & 0,144 & 1 & & & & & & & & \\
\hline & TDS & 0,318 & 0,233 & 0,178 & 0,979 & 1 & & & & & & & \\
\hline & $\mathrm{O} 2$ & 0,337 & 0.175 & $-0,432$ & 0,000 & $-0,023$ & 1 & & & & & & \\
\hline & Transp. & $-0,421$ & .0 .480 & 0,116 & $-0,369$ & $-0,316$ & 0,293 & 1 & & & & & \\
\hline & Prof & 0,245 & 0,263 & $-0,284$ & 0,251 & 0,273 & $-0,171$ & $-0,851$ & 1 & & & & \\
\hline & Pluvio. & 0,531 & 0.542 & -0.094 & 0.370 & 0,335 & $-0,150$ & $-0,894$ & 0,878 & 1 & & & \\
\hline & $\mathrm{PO} 4$ & 0,236 & 0.099 & -0.645 & 0,276 & 0.316 & 0,278 & $-0,164$ & 0.462 & 0,323 & 1 & & \\
\hline & $\mathrm{Ca} 2+$ & $-0,188$ & 0,063 & $-0,245$ & $-0,301$ & $-0,235$ & $-0,035$ & $-0,073$ & 0,051 & -0.109 & $-0,145$ & 1 & \\
\hline & DT & $-0,310$ & $-0,277$ & $-0,525$ & $-0,256$ & $-0,273$ & 0,015 & 0.024 & $-0,137$ & -0.363 & 0,048 & 0,593 & 1 \\
\hline
\end{tabular}



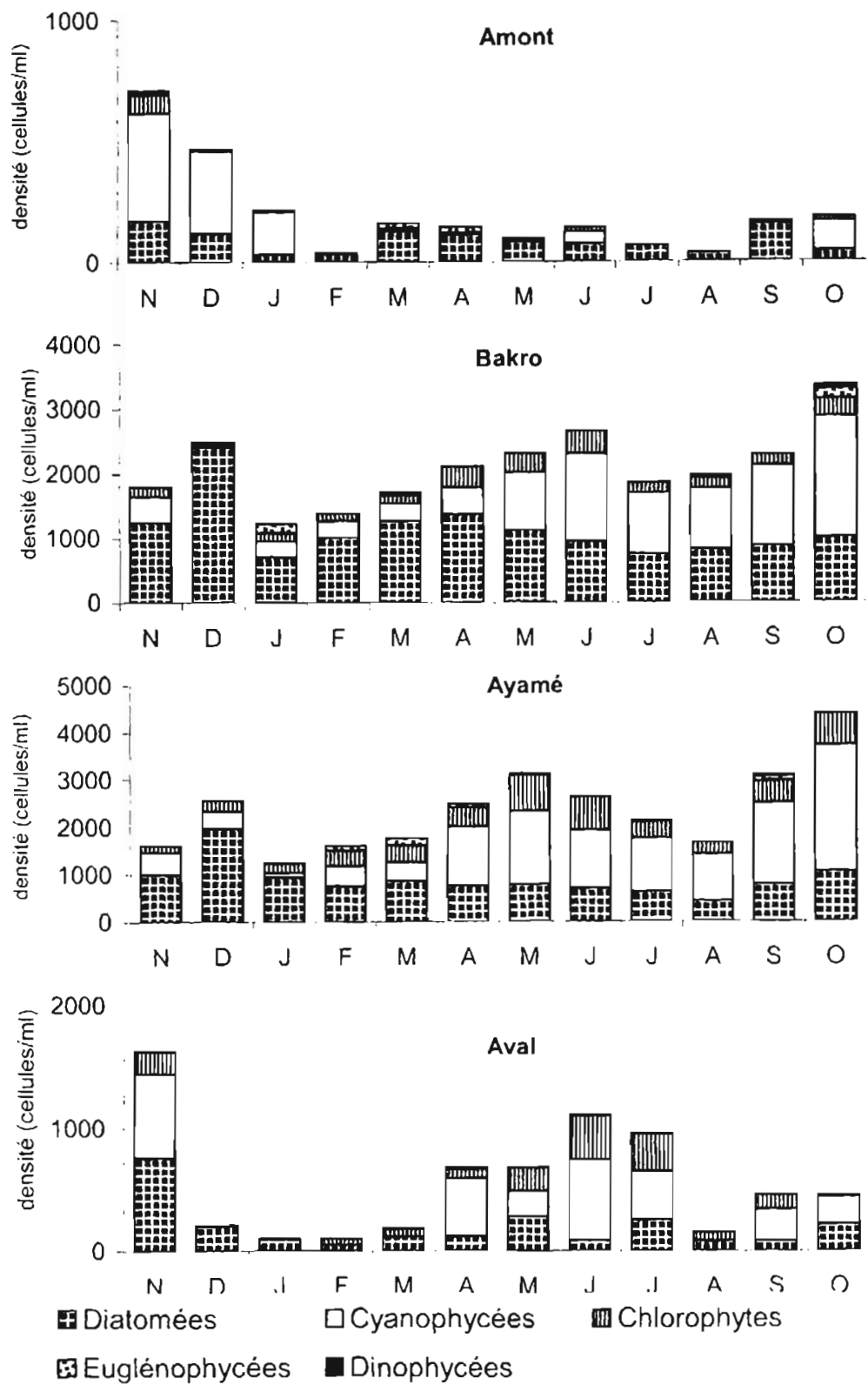

Fig. 4 - Variations des densités des grands groupes phytoplanctoniques en divers endroits de la rivière Bia (amont, Bakro, Ayamé, aval).

Fig. 4 - Distribution of phytoplankton densities along the Bia river (upstream, Ayamé, Bakro, downstream). 
En ce qui concerne la composition taxinomique des peuplements, de février à septembre, les Diatomées (Fragilaria virescens et Aulacoseira granulata) représentent la composante majeure du phytoplancton examiné, constituant même $100 \%$ du peuplement en juillet et août. Durant cette même période, les Cyanophycées sont rares, parfois absentes. Leur développement a lieu de novembre à janvier et surtout en octobre $(82 \%)$. Les Chlorophytes (maximum $18 \%$ en juin), les Euglénophycées (maximum $17 \%$ en avril) et les Dinophycées (maximum $2 \%$ en novembre) ont une faible contribution à l'élévation des densités absolues.

L'évolution de la densité phytoplanctonique dans les deux stations du lac (Bakro et Ayamé) présente à peu près la même allure (fig. 4). Les maximums de densités sont observés en décembre et en octobre dans les deux stations. Des valeurs élevées de la densité sont également notées en mai à Ayamé et juin à Bakro. Les plus faibles densités sont enregistrées en janvier, aussi bien à Bakro qu'à Ayamé, et également en août à Ayamé.

Relativement aux grands groupes phytoplanctoniques, on observe au début de la saison sèche (décembre), la dominance très marquée des Diatomées à Bakro $(97 \%)$ et à Ayamé (76\%). Les espèces Asterionella formosa, Aulacoseira granulata et sa variété angustissima participent à cette poussée phytoplanctonique. Au-delà de cette période, ce groupe voit sa densité diminuer notablement au pro- fit des Cyanophycées qui présentent des densités relativement élevées en mai à Ayamé $(50 \%)$ et en juin à $\mathrm{Ba}-$ kro $(51 \%)$ d'une part et d'autre part en octobre à Ayamé $(60 \%)$ et Bakro (54\%). Pendant ces périodes, on note un développement de certaines espèces comme Anabeana spiroides et Microcystis aeruginosa. Les Chlorophytes évoluent à peu près de la même manière que les Cyanophycées, mais toujours en quantité plus faible. Elles apparaissent relativement importantes en mai à Ayamé $(24 \%)$ et en juin à Bakro (13\%). A part ces trois groupes, qui constituent l'essentiel des populations, on note que les Euglénophycées atteignent près de $12 \%$ en janvier à Bakro et $8 \%$ en mars à Ayamé et les Dinophycées 2,5\% en août à Bakro et $1 \%$ à Ayamé en septembre.

En aval de la rivière Bia, les minimums de densité phytoplanctonique sont enregistrés pendant les périodes de grande saison sèche (décembre mars) et de petite saison sèche (août). Les fortes densités ont été observées en juin et surtout en novembre (fig. 4). Durant les mois de décembre à mars, les Diatomées restent le groupe le plus important du peuplement examiné. Pendant la période indiquée, les Cyanophycées sont absentes. Toutefois, elles sont bien représentées en avril (69\%), en juin $(59 \%)$ et en septembre (57\%). II en est de même avec les Chlorophytes mais à un degré moindre. 


\section{DISCUSSION}

Dans l'ensemble, les densités phytoplanctoniques sont relativement importantes dans le lac (Bakro, Ayamé), peu élevées en aval et faibles en amont.

Dans l'axe longitudinal amont-aval, le phytoplancton se distribue différemment. En effet, le peuplement se modifie avec essentiellement une addition d'espèces et peu de disparition, du moins dans la portion lacustre de la rivière Bia (Ouattara et al., 2000).

Dans le système fluvial, à écoulement plus ou moins rapide et turbulent, les algues phytoplanctoniques sont très peu abondantes. En revanche dans le système lacustre, les densités sont relativement élevées. En effet, les communautés phytoplanctoniques y trouvent des conditions de développement favorables, en liaison avec l'état stagnant des eaux et de leur éclaircissement $(0,8<$ transparence $<1,5 \mathrm{~m}$ ).

Par ailleurs, l'évolution des densités phytoplanctoniques dans le lac (Bakro et Ayamé) met en évidence une périodicité saisonnière du développement des algues. Les populations phytoplanctoniques en saison de pluies (avril - juin et octobre) sont beaucoup plus importantes qu'elles ne le sont en saison sèche (janvier février).

Le cycle annuel de la densité phytoplanctonique en aval des barrages est proche de celui obtenu dans les stations du lac, à cela près que la saison de faible densité phytoplanctonique se situe de janvier à février et en août, la période de densité relativement élevée d'avril à juillet. Cette ressemblance dans l'évolution des densités pourrait s'expliquer par l'influence du lac sur la partie aval de la rivière à travers l'ouverture des vannes des barrages d'Ayamé l et II.

Relativement à la composition phytoplanctonique, les Cyanophycées dominent les peuplements dans les deux stations du lac en saison de pluies et les Diatomées en saison sèche. Ces résultats enregistrés dans le lac d'Ayamé I sont proches de ceux obtenus par Thomas et Ratcliffe (1973) dans le lac Volta au Ghana.

L'importance des Cyanophycées, notamment Microcystis aeruginosa et Anabaena spiroides dans le lac (Bakro et Ayamé) en saison humide, pourrait indiquer que la dynamique de ces espèces est favorisée par la montée des eaux. Cette élévation du niveau des eaux est due principalement à l'arrivée des eaux de ruissellement chargées de produits de lessivage des sols.

Fig. 5 - Ordination en RDA des taxons phytoplanctoniques et des variables physico-chimiques en divers endroits de la rivière Bia au cours de 12 mois : amont $=A m$, Ayamé $=A y, B a k r o=B a, a v a l=$ Av.

Fig. 5 - RDA ordination of phytoplankton taxa and physical and chemical parameters along the Bia river : upstream (Am), Ayamé (Ay), Bakro (Ba), downstream (Av) during 12 months. 

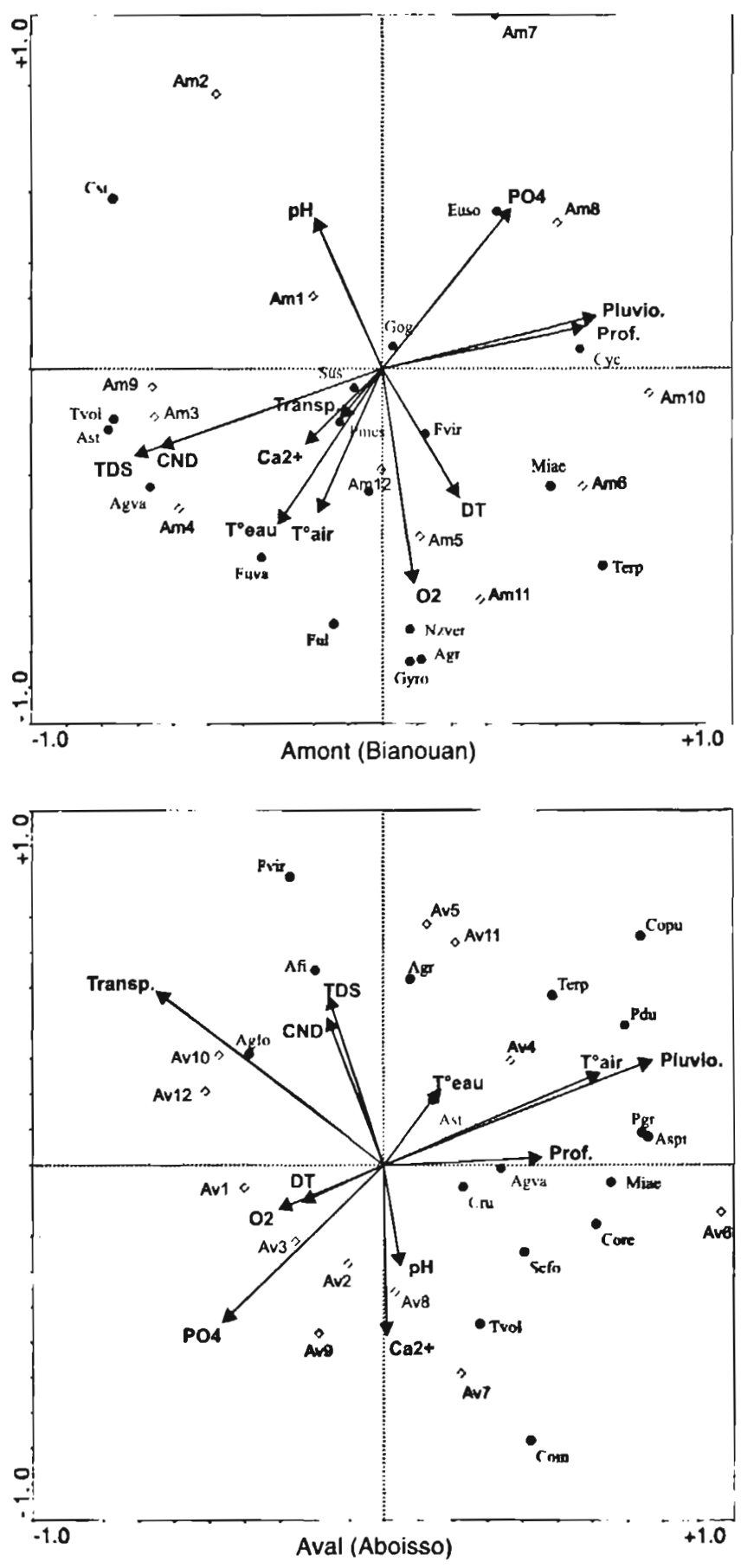

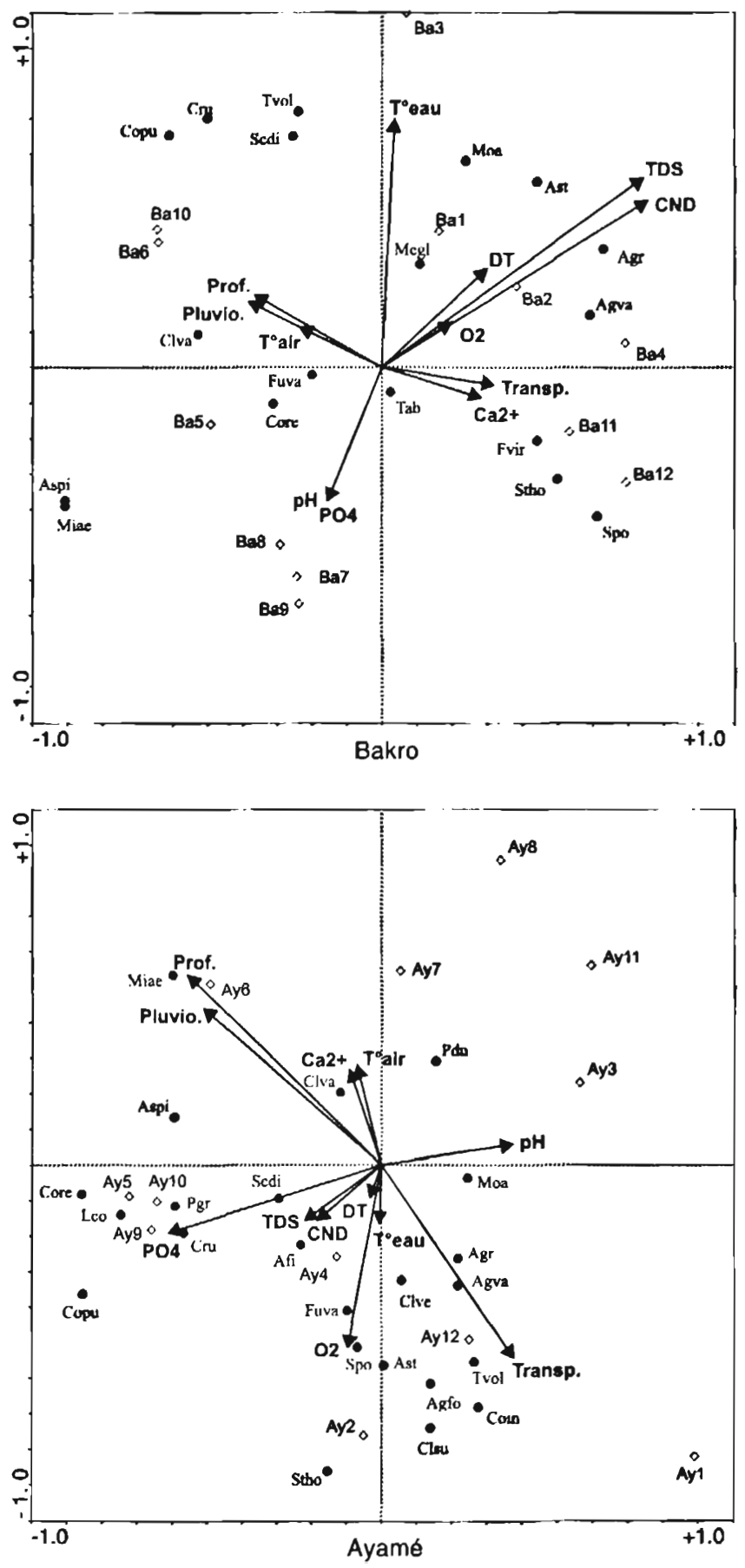
Contrairement au lac et à l'aval des barrages, la dynamique des populations phytoplanctoniques en amont des barrages est différente. Les Diatomées sont majoritaires dans la communauté phytoplanctonique en saison humide. Les proportions relativement importantes des Diatomées dans les peuplements en saison de pluies pourraient être le fait de nanoplancton le plus souvent représenté par des Diatomées de dérive. En effet, la submersion de divers substrats de la zone littorale (végétation et rochers) du bassin lors de la montée des eaux, entraîne le détachement et la mise en suspension de certaines algues par le courant comme Gomphonema gracile et Gyrosigma acuminatum (Cocquyt, 1998). Cette relative dominance des Diatomées de dérive dans la composition phytoplanctonique est également apparue dans les peuplements de certaines rivières du nord de la Côte d'Ivoire notamment Bagoé et Léraba (Iltis, 1982).

Le changement de niveau des eaux suffit à agir sur la densité des organismes qui y vivent. Les variations saisonnières du phytoplancton semblent être liées aux conditions hydrologiques comme l'a souligné Talling (1986) dans la synthèse des travaux réalisés sur certains lacs africains.

L'analyse en RDA (fig. 5) révèle une dynamique complexe du peuplement algal.

Les graphiques permettent de voir dans le plan constitué par les deux axes majeurs que les périodes de fortes densités phytoplanctoniques ne sont pas associées à une saturation en oxygène quelle que soit la station. Les nitrates, nitrites et ammonium contenus dans les eaux des différentes stations montrent une faible variation au cours de la période d'échantillonnage, de sorte qu'ils n'ont pas été affichés par l'analyse dans les différentes ordinations en RDA. Les variations quantitatives du phytoplancton, rapprochées des valeurs de phosphates, ne fournissent pas d'indications quant à leur interprétation en amont et en aval du barrage (fig. $5 \mathrm{~A}$ et $5 \mathrm{~B}$ ). De même, le carré de corrélation extrait de la RDA sur la période d'étude dans les stations du lac (Bakro et Ayamé), n'illustre pas nettement l'influence de l'activité photosynthétique: P-PO4, consommé en période de développement phytoplanctonique ne s'oppose pas parfaitement à $\mathrm{O}_{2}$ et au pH auxquels est associée la biomasse algale (fig. 5C et 5D).

D'autres facteurs pourraient être pris en compte dans les fluctuations des densités phytoplanctoniques notamment dans les stations du lac d'Ayamé I. En effet, les variations sajsonnières du zooplancton observées (Nobah, 1998) au cours de notre période d'étude montrent que le maximum de zooplancton a été enregistré en grande saison sèche (janvier-février), période du minimum du phytoplancton. De plus, Koné (2000) a montré que l'espèce Sarotherodon melanotheron consomme en majorité le phytoplancton dans le lac d'Ayamé $\mathrm{l}$. Ces phénomènes de broutage pourraient être déterminants dans les 
fluctuations de densité observées au niveau du phytoplancton du lac.

\section{CONCLUSION}

Les variations saisonnières de la densité phytoplanctonique en rapport avec les paramètres physico-chimiques paraissent ici difficiles à interpréter dans chaque station. Ces variations phytoplanctoniques dans les différents milieux semblent dépendre des conditions hydrologiques.

Les facteurs du milieu n'étant pas identiques dans toutes les stations étudiées, les densités phytoplanctoniques relativement importantes dans le lac d'Ayamé I, peu élevées en aval et faibles en amont des barrages hydroélectriques reflètent l'hétérogénéité des biotopes.

D'autres investigations sont nécessaires en vue de mieux distinguer la variabilité phytoplanctonique naturelle de celle pouvant relever des barrages et de leur fonctionnement.

\section{REMERCIEMENTS}

$\mathrm{Ce}$ travail fait partie du projet V.L.I.R/K.U.L (Vlaamse Interuniversitaire Raad) "Évolution de la biodiversité des poissons après la construction d'un barrage hydroélectrique: Cas de la rivière Bia en Côte d'Ivoire ", financé par l'Administration Générale pour la Coopération au developpement (AGCD-ABOS) de la Belgique.

Nous tenons à remercier les promoteurs du projet : D.F.E. Thys van den Audenaerde et G.G. Teugels.

\section{RÉFÉRENCES BIBLIOGRAPHIQUES}

Carlson R.E., 1977. A trophic state index for lakes. Limnol. Oceanogr. 22 (2): 361-369.

Cocquyt C., 1998. Dialoms from the Northern Basin of Lake Tanganyika. Biblio. Diatomolo. Band 39, édition J. Cramer, $274 \mathrm{p}$.

Dufour P., Durand J.R., 1982. La production végétale des lagunes de Côte d'Ivoire. Rev. Hydrobiol. trop. 15 (3): 209-230.

Gourène G., Teugels G.G., Hugueny B., Thys Van Den Audenaerde D.F.E., 1999. Evaluation de la diversité ichtyologique d'un bassin ouest africain après la construction d'un barrage. Cybium, 23 (2) : 147-160.

Iltis A., 1982. Peuplements algaux des rivières de Côte d'Ivoire. II - Variations saisonnières des biovolumes, de la composition et de la diversité spécifique. Rev. Hydrobiol. trop., 15 (3) : 241-251.

Koné T., 2000. Régime alimentaire et reproduction d'un tilapia lagunaire ( $\mathrm{Sa}$ rotherodon melanotheron, Rüppell, 1852) dans des conditions fluviatiles et lacustres (rivière Bia et lac d'Ayamé). Thèse de doctorat, Katholieke Universiteif Leuven, 259 p.

Lazzaro X., 1981. Biomasses, peuplements phytoplanctoniques et production primaire du lac Titicaca. Rev. Hydrobiol. trop., 14 (4) : 349-380.

Muylaert K., Van Kerckvoorde A., Vyverman W., Sabbe K., 1997. Structural characteristics of phytoplankton assemblages in tidal and non-tidal freshwater systems: a case-study from the Schelde basin. Freshwat. Biol., (38) : 263-276.

Nobah C.S.H., 1998. Distribution verticale des peuplements zooplanctoniques dans un lac de barrage Ouest africain : lac d'Ayamé I (Côte d'Ivoire). D.E.A., Université de Cocody, 65 p. 
Ouattara A., Podoor N., Teugels G.G., Gourène G., 2000. Les micro-algues de deux cours d'eau (Bia, Agnébi) de Côte d'Ivoire. Systematic and Geography of plant, 70 (2) : 46-70.

Reizer C., 1967. Aménagement piscicole du lac d'Ayamé. CTFT, $46 \mathrm{p}$.

Talling J.F., 1986. The seasonality of phytoplankton in Africa lakes. Hydrobiologia (138) : 139-160.

Ter Braak C.J.F., Smilauer P., 1998. CANOCO Reference manual and user's guide to Canoco for Windows (version 4). Center for Biometry Wageningen, $351 \mathrm{p}$.

Thomas J.D., Ratcliffe P.J., 1973. Observations on the limnology and primary production of a small man-made lake in the West African savanna. Freshwat. Biol., (3) : 573-612.

Uehlinger V., 1964. Étude statistique des méthodes de dénombrement planctonique. Arch. Sci. Univ. Genève, (17) : 121-223.

Van den Bossche J.P., Bernacsek G.M., 1990. Source book for the inland fishery resources of Africa. FAO Fischeries Technical Paper, 18/2, Rome, 240 p.

Van Tongeren O.F.R., Van Liere L., Gulati R.D., Postema G., Boesewinkel-De Bruyn P.J., 1992. Multivariate analysis of the plankton communities in the Loosdrecht lakes: relationship with the chemical and physical environment. Hydrobiologia, (233) : 105-117.

\section{Liste des acronymes des espèces}

Anabaena affinis (Afi), Anabaena spiroides (Aspi), Asterionella formosa (Ast), Aulacoseira granulata (Agr), Aulacoseira granulata var. angustissima (Agva), Aulacoseira granulata var. angustissima fo. spiralis (Agfo), Closterium acutum var. variabile (Clva), Closterium subulatum var. subulatum (Clsu), Closterium venus (Clve), Coelastrum indicum (Coin), Coelastrum reticulatum (Core), Coelomoron pusillum (Copu), Crucigeniella crucifera (Cru), Cyclotella meneghiniana (Cyc), Cymbella silesiaca (Csi), Euastrum binale (Ebi), Eunotia soleirolii (Euso), Fragilaria ulna (Ful), Fragilaria ulna var. acus (Fuva), Fragilaria virescens (Fvir), Gomphonema gracile (Gog), Gyrosigma acuminatum (Gyro), Lyngbya contorta (LCo), Merismopedia glauca (Megl), Microcystis aeruginosa (Miae), Monoraphidium arcuatum (Moa), Navicula elginensis var. elginensis (Nel), Nitzschia vermicularis (Nzver), Pediastrum duplex var. duplex (Pdu), Pediastrum duplex var. gracillimum (Pgr), Peridinium cinctum (Peri), Pinnularia mesolepta (Pmes), Plectonema gracillimum (Plec), Scenedesmus acutiformis (Scac), Scenedesmus disciformis (Scdi), Staurastrum polymorphum (Spo), Staurastrum thomassonianum (Stho), Tabellaria flocculosa (Tab), Terpsinoe musica (Terp), Trachelomonas volvocina (Tvol). 